\title{
Transtornos MENTAIS COMUNS E CONSUMO DE BEBIDA ALCOÓliCA E TABACO ENTRE ESTUDANTES DE ENFERMAGEM DE UMA UNIVERSIDADE PÚBLICA NA Amazônia Ocidental brasileira
}

\section{Bruno Pereira da Silva \\ Clarissa Mendonça Corradi-Webster ${ }^{2}$ Edilaine Cristina da Silva Gherardi Donato ${ }^{3}$ Miyeko Hayashida ${ }^{4}$ Marluce Miguel de Siqueira ${ }^{5}$}

O objetivo deste estudo é identificar a prevalência de transtornos mentais comuns e consumo de bebida alcoólica e tabaco entre estudantes de enfermagem de uma universidade pública da Amazônia Ocidental brasileira. Trata-se de um estudo exploratório e descritivo, utilizando-se os instrumentos AUDIT, SRQ-20 e questionário de tabagismo. Do total de estudantes, 93,4\% afirmou fazer consumo de álcool de baixo risco, porém $26 \%$ afirmou beber no padrão binge pelo menos uma vez ao mês. Em relação ao tabaco, 78,6\% afirmou ter usado por curiosidade e desejo de experimentar e 46,1\% afirmou que "sente-se nervoso, tenso ou preocupado" e que tem "humor depressivo/ansioso". A comparação dos resultados possibilita o planejamento de programas de prevenção mais condizentes com as características e necessidade de cada comunidade.

Descritores: Alcoolismo; Hábito de Fumar; Diagnóstico Duplo (Psiquiatria); Estudantes de Enfermagem.

\footnotetext{
${ }^{1}$ MSc, Professor Assistente, Centro Multidisciplinar, Universidade Federal do Acre, Campus Floresta, Cruzeiro do Sul, AC, Brasil.

2 PhD, Professor Doutor, Faculdade de Filosofia, Ciências e Letras de Ribeirão Preto, Universidade de São Paulo, Ribeirão Preto, SP, Brasil.

${ }^{3} \mathrm{PhD}$, Professor Associado, Escola de Enfermagem de Ribeirão Preto, Universidade de São Paulo, Centro Colaborador da OMS para o Desenvolvimento da Pesquisa em Enfermagem, Ribeirão Preto, SP, Brasil.

${ }^{4}$ PhD, Enfermeira, Escola de Enfermagem de Ribeirão Preto, Universidade de São Paulo, Centro Colaborador da OMS para o Desenvolvimento da Pesquisa em Enfermagem, Ribeirão Preto, SP, Brasil.

${ }^{5} \mathrm{PhD}$, Professor Associado, Universidade Federal do Espírito Santo, Vitória, ES, Brasil.
} 


\section{COMMON MENTAL DISORDERS, ALCOHOL CONSUMPTION AND TOBACCO}

\section{USE, AMONG NURSING STUDENTS AT A PUBLIC UNIVERSITY IN THE WESTERN Brazilian Amazon}

The objective of this study is to identify the prevalence of two common mental disorders, alcohol consumption and tobacco use, among nursing students of a public university in the western Brazilian Amazon. This is an exploratory and descriptive study, using AUDIT instruments, SRQ-20 and smoking questionnaire. Of the total number of students $93.4 \%$ said they consume a low-risk amount of alcohol, but 26\% said they binge drank at least once a month. As for tobacco, $78.6 \%$ claimed to have used it because of curiosity and the desire to experiment and $46.1 \%$ said they "felt nervous, tense or worried" and had a "depressive/anxious mood." In comparing these results it allows for a more consistent planning of prevention programs that addresses the needs of this community.

Descriptors: Alcoholism; Smoking; Diagnosis, Dual (Psychiatry); Students, Nursing.

\section{Trastornos Mentales COMUNES Y CONSUMO DE BEBIDA ALCOHÓliCA Y TABACO ENTRE ESTUDIANTES DE ENFERMERÍA DE UNA UNIVERSIDAD PÚBLICA EN la Amazonía OCCidental brasileña}

El objetivo de este estudio es identificar la prevalencia de trastornos mentales comunes y consumo de bebida alcohólica y tabaco entre estudiantes de enfermería de una universidad pública de la Amazonía Occidental brasileña. Se Trata de un estudio exploratorio y descriptivo, utilizándose los instrumentos AUDIT, SRQ-20 y el cuestionario de tabaquismo. Del total de estudiantes, 93,4\% afirmó hacer consumo de alcohol de bajo riesgo, pero el $26 \%$ afirmó beber en el modelo binge por lo menos una vez al mes. En relación al tabaco, 78,6\% afirmó haber usado por curiosidad y deseo de experimentar el $46,1 \%$ afirmó que "se siente nervioso, tenso o preocupado" y que tiene "humor depresivo/ ansioso". La comparación de los resultados posibilita el planeamiento de programas de prevención más coincidentes con las características y necesidades de cada comunidad.

Descriptores: Alcoholismo; Hábito de Fumar; Diagnóstico Dual (Psiquiatría); Estudantes de Enfermería.

\section{Introdução}

A literatura aponta que os sintomas de ansiedade e depressão ${ }^{(1-2)}$ e o consumo abusivo de substâncias psicoativas vêm crescendo entre os jovens ${ }^{(1-4)}$. A população universitária é um grupo importante de ser estudada epidemiologicamente. São geralmente pessoas que estão na transição da adolescência para a idade adulta, tendo que lidar com as diferentes tarefas desta etapa do desenvolvimento; tarefas que podem ser geradoras de sofrimento emocional. Entre elas, destacam-se o desenvolvimento de novas relações e repertórios sociais, o afastamento emocional e físico da família de origem, a construção da autonomia e de valores e o desenvolvimento de novos papéis sociais $^{(3)}$. 
Além disso, as Instituições de Ensino Superior (IES) estão cada vez mais competitivas. Tal competitividade tem início já no processo seletivo do vestibular, que muitas vezes é concorrido para o ingresso em uma boa universidade. Uma vez na universidade, existem as demandas acadêmicas de cada curso, bem como as expectativas para ingressar no mercado de trabalho ${ }^{(5)}$.

Entre os jovens universitários da área da Saúde, esse quadro é agravado pelo contato emocional próximo com diferentes pessoas e com a dor destas. Os estudantes são geralmente pessoas que fizeram a escolha de ajudar outros seres humanos a nascerem, vivenciarem (superar os problemas e as limitações) e morrerem dignamente ${ }^{(6-7)}$.

Alguns estudos já abordaram a temática saúde mental entre universitários. Em estudo desenvolvido com estudantes da área da Saúde de uma universidade pública no interior do estado de São Paulo, foi encontrada a prevalência de $23 \%$ de uso problemático de álcool (UPA), $30,6 \%$ de transtornos mentais comuns (TMC) e $7,3 \%$ de coocorrência de UPA e $\mathrm{TMC}^{(4)}$. Em outro estudo, realizado com estudantes de dois cursos de graduação em Enfermagem, verificou-se que uma taxa de prevalência de sintomas indicativos de depressão entre os entrevistados de $14 \%(n=114)^{(2)}$. Resultados expressivos foram encontrados em uma pesquisa que investigou os sintomas depressivos entre alunos de Medicina de uma universidade pública, cuja prevalência foi de 40,5\% $(n=84)$. Desses, $1,2 \%$ apresentou sintomas depressivos graves, $4,8 \%$, moderados e $34,5 \%$, leves ${ }^{(1)}$.

Tendo em vista a relevância da temática e o impacto que o consumo indevido de substâncias psicoativas pode ter para jovens que se preparam para a vida adulta e para o mercado de trabalho, alguns estudos têm tratado especificamente do assunto. Alguns abordam estudantes da área da Saúde, em especial os acadêmicos de Medicina ${ }^{(8-9)}$, Enfermagem ${ }^{(10-11)}$ e, em menor quantidade, Farmácia ${ }^{(12)}$ e Odontologia ${ }^{(13)}$. Corroborando com a presente argumentação sobre a importância da temática, em 2010, a Secretária Nacional de Políticas Sobre Drogas, publicou o I Levantamento Nacional sobre o Uso de Álcool, Tabaco e outras Drogas entre Universitários das 27 Capitais Brasileiras ${ }^{5}$. Esse levantamento pesquisou 12.856 universitários de 100 instituições, sendo 51 públicas e 49 privadas e mostrou o perfil do uso de SPAs dos universitários brasileiros. Os resultados mostraram que o consumo de álcool de risco baixo, moderado e alto por universitários foi de 78,2\%, 19,2\% e $2,6 \%$, respectivamente. O consumo de álcool no padrão binge drinking representou $37,7 \%$ e $25,3 \%$ de consumo pesado nos últimos 12 meses e 30 dias, respectivamente. O tabaco foi a segunda SPA mais utilizada, com 46,7\% para uso na vida, $27,8 \%$ no ano e $21,6 \%$ no mês. $\mathrm{Na}$ região Norte do país, o consumo de álcool de risco baixo, moderado e alto por universitários foi de $79,2 \%, 18,9 \%$ e $1,9 \%$, respectivamente, e $39,4 \%$ e $31,4 \%$ deles fizeram o consumo de álcool no padrão binge drinking. Para o tabaco, o uso na vida e no ano foi de $17,9 \%$, e no mês foi de 14,1\%. Esses estudos desempenham um papel importante para sociedade brasileira, uma vez que reforçam a necessidade da abordagem da temática nas universidades, preocupando-se com a saúde mental dos jovens estudantes de graduação, principalmente os inseridos na área da Saúde ${ }^{(2)}$.

Todavia, os estudos existentes, em sua maioria, trazem informações de amostras de universitários pertencentes a IES de algumas regiões do país, principalmente do Sudeste, com ênfase no Estado de São Paulo. Tal situação limita a comparação de resultados, bem como a construção de uma realidade nacional e fidedigna ${ }^{(5)}$. A realidade sobre o UPA, o consumo de tabaco e a prevalência dos TMC e os efeitos da associação do uso dessas SPAs com as morbidades psiquiátricas entre estudantes da região Norte do país foi pouco estudada ${ }^{(8)}$.

Mediante o exposto faz-se necessário analisar os fatores associados à ocorrência TMC, ao consumo de álcool e tabaco entre os universitários. Tal análise é extremamente importante para evitar o agravamento da sintomatologia e o desenvolvimento de um quadro clínico psicopatológico, além de possibilitar o desenvolvimento de estratégias de redução de danos relacionados ao consumo de substâncias.

Dessa forma, este estudo tem como objetivo identificar a prevalência de transtornos mentais comuns e de consumo de álcool e tabaco entre os universitários do curso de 
graduação em Enfermagem do Campus Floresta da Universidade Federal do Acre (UFAC). O presente estudo poderá contribuir na compreensão e no planejamento de medidas preventivas em saúde adequadas no meio acadêmico.

\section{Métodos}

Foi realizado um estudo quantitativo, exploratório e descritivo. O cenário do estudo foi o Centro Multidisciplinar, Campus Floresta da Universidade Federal do Acre. A coleta foi realizada no mês de maio de 2012, com autorização prévia dos professores e da instituição.

Os participantes foram 76 estudantes uni-

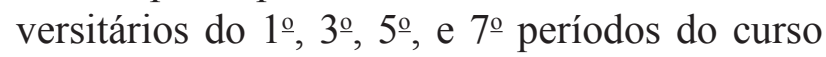
de graduação em Enfermagem dessa instituição. Assim, os critérios de inclusão foram: ser aluno do curso de Enfermagem, estar matriculado no 1을 semestre letivo de 2012 e concordar em participar do estudo.

A coleta dos dados realizou-se por meio da utilização de três instrumentos autoaplicáveis; destes, dois de domínio público. O primeiro instrumento foi o Alcohol Use Disorder Identification Test (AUDIT) $)^{(14)}$ validado para o contexto brasileiro e utilizado para rastrear padrões de consumo de álcool. Suas perguntas avaliam o padrão de consumo de álcool, os sinais e sintomas da dependência e os problemas decorrentes do uso dessa substância. O segundo instrumento foi um questionário autoaplicável com questões fechadas, desenvolvido e validado por pesquisadores ${ }^{(11)}$ da Universidade Estadual do Rio de Janeiro (UERJ), para investigar o uso do tabaco entre os universitários do curso de Enfermagem. Este investiga variáveis sociodemográficas e questões relacionadas ao consumo do tabaco. O terceiro questionário foi o Self Reporting Questionnaire (SRQ-20), instrumento utilizado para o rastreamento de morbidades psiquiátricas menores, transtornos não psicóticos e/ou transtornos mentais comuns (TMC). Apresenta 20 questões, com respostas do tipo sim ou não. Respostas positivas indicam maior intensidade de TMC ou sofrimento emocional. Escores acima de seis indicam caso clínico positivo para $\mathrm{TMC}^{(15)}$.

Os instrumentos foram aplicados em sala de aula com a permissão do professor que ministrava aula no momento e autorização prévia da coordenação do curso de Enfermagem. O tempo médio para responder aos questionários foi de 40 minutos.

Os dados foram inseridos e organizados em uma planilha do programa Microsoft Excel. Utilizou-se a estatística descritiva com emprego da frequência absoluta e percentual para análise dos dados tratados no pacote estatístico Statistical Package for the Social Science - SPSS 17.

A pesquisa foi submetida e aprovada pelo Comitê de Ética em Pesquisa (CEP) da UFAC. Os participantes foram informados dos objetivos da pesquisa e de seus direitos quanto à participação, respeitando a Resolução no 466/2012 do Conselho Nacional de Saúde ${ }^{(16)}$. Foi também solicitado que assinassem o Termo de Consentimento Livre e Esclarecido.

\section{Resultados}

A população do estudo foi composta por 120 estudantes do curso de graduação de Enfermagem, desses, 76 alunos estavam presentes em sala de aula no momento da aplicação dos instrumentos, abrangendo todas as turmas de Enfermagem da instituição. Assim, 76 sujeitos assinaram o Termo de Consentimento Livre e Esclarecido, concordando em participar da pesquisa. As perdas se deram pela ausência; não houve recusas. A maioria era mulher $(73,37 \%$, $\mathrm{n}=56)$, com idade até 25 anos $(76,1 \%, \mathrm{n}=57)$ e solteira $(77,6 \%, \mathrm{n}=59)$.

A aplicação do AUDIT foi utilizada para a identificação de uso problemático de álcool (UPA) com ponto de corte sete. A análise do escore total do AUDIT da amostra de 76 universitários identificou que $5(6,6 \%)$ estudantes apresentam AUDIT positivo (escore maior ou igual a 8), identificando uso problemático de álcool, 35 (46,1\%) estudantes apresentaram AUDIT negativo (escore menor ou igual a 7), identificando uso de baixo risco de álcool, incluindo $36(47,3 \%)$ que informaram não ter consumido bebidas alcoólicas nos últimos 12 meses.

Entre os acadêmicos de Enfermagem, 93,4\% apresentou um padrão de beber (Tabela 1) na zona de risco I do AUDIT (escore maior ou igual a 0 e menor ou igual a 7 pontos), caracterizando uso de baixo de risco de álcool, incluindo os 
estudantes que informaram não ter consumido bebida alcoólica nos últimos 12 meses. Já na zona II do AUDIT (escore maior ou igual a $8 \mathrm{e}$ menor ou igual a 15 pontos), foi identificado que $6,6 \%$ apresentou um padrão de beber característico de uso de risco de álcool.

No presente estudo, não foram identificados estudantes com padrão característico de uso nocivo de álcool nem de uma provável síndrome de dependência do álcool, zona III e IV do AUDIT (escore maior ou igual a 16 e menor ou igual a 20 pontos e, maior que 20 pontos, respectivamente).

Tabela 1 - Distribuição do padrão de beber dos estudantes de Enfermagem de uma universidade pública, segundo as zonas de risco do AUDIT. Cruzeiro do Sul-AC, Brasil, 2012

\begin{tabular}{lcc}
\hline Zonas do AUDIT & N & $\%$ \\
\hline Zona I & 71 & 93,4 \\
Zona II & 5 & 6,6 \\
Zona III & 0 & 0,0 \\
Zona IV & 0 & 0,0 \\
\hline Total & 76 & 100 \\
\hline
\end{tabular}

Sobre o padrão de binge drinking (questões 2 e 3 do AUDIT), 4 (5,4\%) estudantes mencionaram beber duas ou três doses de álcool em um dia típico e $3(4,1 \%)$, seis ou sete doses. Já sobre a frequência de cinco ou mais doses de álcool em uma ocasião, $19(26,0 \%)$ beberam menos que uma vez por mês, $6(8,2 \%)$ uma vez por mês e 2 $(2,7 \%)$ uma vez por semana.

A experiência dos universitários com o tabaco é apresentada na Tabela 2. Observou-se que $28(37,3 \%)$ dos entrevistados fumaram alguma vez na vida. Desses que experimentaram, apenas um continua fazendo o uso do tabaco.

Tabela 2 - Experiência dos estudantes de Enfermagem de uma universidade pública com o uso do tabaco. Cruzeiro do Sul-AC, Brasil, 2012

\begin{tabular}{lcc}
\hline Características & $\mathbf{N}$ & $\%$ \\
\hline Experimentou fumar alguma vez na vida & & \\
Sim & 28 & 37,3 \\
Não & 47 & 62,7 \\
Total & 75 & 100 \\
Se já experimentou, continua usando & & \\
Sim & 1 & 3,6 \\
Não & 27 & 96,4 \\
Total & 28 & 100 \\
\hline
\end{tabular}

A Tabela 3 apresenta os motivos que levaram os estudantes ao uso do tabaco. Observa-se que $78,6 \%$ fez uso por curiosidade e desejo de experimentar, $21,4 \%$ por influência do grupo extrafamiliar e $10,7 \%$ por desconhecimento dos prejuízos à saúde.

Tabela 3 - Motivos que levaram os estudantes de Enfermagem de uma universidade pública ao uso do tabaco $(n=31)$. Cruzeiro do Sul-AC, Brasil, 2012

\begin{tabular}{lcc}
\hline Motivos & N & $\%$ \\
\hline Curiosidade e desejo de experimentar & 22 & 78,6 \\
Influência do grupo extrafamiliar & 6 & 21,4 \\
Desconhecimento do prejuízo à saúde & 3 & 10,7 \\
\hline
\end{tabular}

O resultado do SRQ 20 foi distribuído pelos grupos de sintomas reagrupados ${ }^{(16)}$. A Tabela 4 apresenta a distribuição das respostas da amostra pelos itens reagrupados do SRQ-20.

Nota-se que no grupo de sintomas "humor depressivo/ansioso", 46,1\% indicou os itens "sente-se nervoso(a), tenso(a) ou preocupado(a)", $31,6 \%$ "assusta-se com facilidade" e 28,9\% "tem se sentido triste ultimamente". No grupo de "sintomas somáticos", 31,6\% tem dores de cabeça frequentes e $25 \%$ "tem sensações desagradáveis no estômago". No grupo de sintomas "decréscimo de energia vital", 32,9\% informou ter "dificuldade para tomar decisões" e 26,3\% que "se cansa com facilidade". No último grupo, nomeado como "Pensamentos depressivos", $9,2 \%$ afirmou que "tem perdido o interesse pelas coisas".

Tabela 4 - Sintomas psiquiátricos menores referidos pelos estudantes de Enfermagem de uma universidade pública $(\mathrm{n}=76)$, Cruzeiro do Sul-AC, Brasil, 2012

\begin{tabular}{lcc}
\hline Grupos de sintomas psiquiátricos menores & N & $\%$ \\
\hline Humor depressivo/ansioso & & \\
Sente-se nervoso(a), tenso(a) ou preocupado(a) & 35 & 46,1 \\
Assusta-se com facilidade & 24 & 31,6 \\
Tem se sentido triste ultimamente & 22 & 28,9 \\
Tem chorado mais do que de costume & 16 & 21,1 \\
Sintomas somáticos & & \\
Tem dores de cabeça frequente & 24 & 31,6 \\
Tem falta de apetite & 12 & 15,8 \\
Dorme mal & 15 & 19,7 \\
Tem má digestão & 19 & 25,0 \\
Tem sensações desagradáveis no estômago & 19 & 25,0 \\
\hline
\end{tabular}


Tabela 4 - Continuação

\begin{tabular}{|c|c|c|}
\hline Grupos de sintomas psiquiátricos menores & $\mathbf{N}$ & $\%$ \\
\hline \multicolumn{3}{|l|}{ Decréscimo de energia vital } \\
\hline Tem dificuldade de pensar com clareza & 13 & 17,1 \\
\hline $\begin{array}{l}\text { Tem/Encontra dificuldades para realizar com } \\
\text { satisfação suas atividades }\end{array}$ & 8 & 10,5 \\
\hline Tem dificuldade para tomar decisões & 25 & 32,9 \\
\hline $\begin{array}{l}\text { Tem dificuldade no serviço (seu trabalho é penoso, } \\
\text { causa sofrimento) }\end{array}$ & 4 & 5,3 \\
\hline Sente-se cansado o tempo todo (a) & 14 & 18,4 \\
\hline Você se cansa com facilidade & 20 & 26,3 \\
\hline \multicolumn{3}{|l|}{ Pensamentos depressivos } \\
\hline $\begin{array}{l}\text { É incapaz de desempenhar um papel útil em sua } \\
\text { vida }\end{array}$ & 1 & 1,3 \\
\hline Tem perdido o interesse pelas coisas & 7 & 9,2 \\
\hline Você se sente uma pessoa inútil, sem préstimos & 1 & 1,3 \\
\hline Tem tido ideia de acabar com a vida & 2 & 2,6 \\
\hline
\end{tabular}

Com a aplicação do teste de qui-quadrado buscou-se conhecer a associação entre transtornos mentais comuns e o consumo de álcool e tabaco. Não foram encontradas associações entre TMC e consumo de bebida alcoólica $\left(\chi^{2}=0,24\right.$; $\mathrm{p}=0,530)$ nem entre TMC e consumo de tabaco $\left(\chi^{2}=1,33 ; p=0,204\right)$.

\section{Discussão}

Em estudo ${ }^{(17)}$ com universitários do curso de Enfermagem de uma instituição de ensino superior privada, o perfil sociodemográfico mostra que a maioria dos universitários é do sexo feminino, solteiro e está na faixa etária de 20 a 25 anos. Esse perfil assemelha-se ao do presente estudo e com outros achados da literatura ${ }^{(10-11)}$.

De acordo com o estudo que investigou a coocorrência de uso problemático de álcool e transtornos mentais comuns em estudantes de graduação da área da Saúde, realizado por Yosetake ${ }^{(4)}$, $76,5 \%$ do estudantes apresentaram um padrão de beber na zona de risco I do AUDIT (consumo de baixo risco), 20,5\% na zona II (consumo de risco), 2,6\% na Zona III (consumo abusivo) e 0,4\% na Zona IV (provável dependência). Vale notar que o estudo em questão também trabalhou com uma amostra majoritariamente feminina, mas com um número maior de homens do que a desta pesquisa, o que poderia explicar os escores maiores encontrados pelo autor.

Em relação ao consumo de bebida alcoólica, pesquisadores $^{(18)}$ encontraram que $83,4 \%$ dos estudantes de duas escolas de Enfermagem a consumiram no último ano. Do total de estudantes,
$73,4 \%$ pontuou AUDIT $\leq 8$. Esses números se aproximam dos encontrados neste estudo.

Sobre a prevalência de episódios de binge drinking, um estudo revelou que $33,4 \%$ dos estudantes bebiam deste modo pelo menos uma vez ao mês ${ }^{(4)}$. No presente estudo, apesar de 94,6\% dos estudantes fazerem consumo de baixo risco, $26 \%$ afirmou consumir bebidas de modo binging pelo menos uma vez ao mês. Considerando-se que o consumo de altas doses de bebidas alcoólicas em uma única ocasião pode colocar os jovens em risco, faz-se importante desenvolver estratégias de redução de danos com esta população ${ }^{(3)}$. Esse dado também indica que, quando utilizado na clínica, o AUDIT deve ser considerado não apenas em relação à sua pontuação total, mas também devem ser observadas as questões individualmente.

Sobre a experimentação do tabaco, encontrou-se na literatura nacional que $23 \%$ dos estudantes de Enfermagem do Centro Universitário Norte do Espírito Santo (CEUNES) ${ }^{(10)}$ experimentaram a substância alguma vez na vida e $77 \%$ deles nunca o fizeram. Entre aqueles que experimentaram, apenas $13 \%$ continuou consumindo tabaco.

Os resultados de uma pesquisa ${ }^{(17)}$ realizada com estudantes de Enfermagem de uma faculdade privada de Guarapari-ES, mostraram que, quando questionados sobre os principais motivos para o uso do tabaco, $33,3 \%$ dos sujeitos responderam tratar-se de curiosidade e desejo de fumar e 19\% por influência do grupo extrafamiliar. Em outra pesquisa com estudantes da mesma área, do CEUNES, dos motivos que levaram os universitários ao hábito de fumar, $75 \%$ estão relacionados à curiosidade e ao desejo de fumar, e $25 \%$ à influência do grupo extrafamiliar ${ }^{(10)}$. Neste estudo, essas questões também foram as mais prevalentes.

De acordo com a literatura ${ }^{(10,17)}$, as motivações encontradas para o consumo de tabaco pelos estudantes universitários são relacionados a fatores do entorno social, como família, meios de comunicação e amigos e também por características pessoais, sentimento de necessidade de pertença, curiosidade, prazer e ociosidade, corroborando os achados deste trabalho.

Os resultados relativos à prevalência de sintomas psiquiátricos menores, rastreado pelo 
SRQ 20, encontrados em estudo realizado com estudantes da Saúde do interior de São Paulo são semelhantes ao deste estudo ${ }^{(4)}$. Em ambos os estudos os sintomas psiquiátricos menores foram reagrupados em "humor depressivo/ansioso", "decréscimo de energia vital", "sintomas somáticos", "pensamentos depressivos". No grupo de "humor depressivo/ansioso" foi observado que $56,5 \%$ informou sentir-se nervoso, tenso ou preocupado, 39,2\% disse que se cansa com facilidade. No grupo denominado "decréscimo de energia vital”, 28,9\% afirmou ter sensações desagradáveis no estômago, já nos grupos de "sintomas somáticos" e de "pensamentos depressivos" $19,8 \%$ "tem perdido o interesse pelas coisas". Corroborando com os resultados apresentados na Tabela 4 deste estudo.

Observou-se em estudo que visou a investigar depressão entre estudantes de dois cursos de Enfermagem de universidade pública do interior de São Paulo ${ }^{(2)}$ que 40,2\% apresentava sinais indicativos de depressão (leve, moderada e grave). Apesar de ter sido encontrado uma alta prevalência de sintomas de transtornos mentais comuns na amostra, neste estudo não foi encontrada associação entre o consumo problemático de álcool e tais sintomas.

\section{Conclusão}

Este estudo investigou a prevalência do uso problemático de álcool, consumo de tabaco e ocorrência de transtornos mentais comuns entre universitários do curso de Enfermagem.

Apesar de muitos estudantes relatarem já ter experimentado bebidas alcoólicas, apenas 5,3\% deles foram apontados como fazendo uso problemático da substância. Esse número pequeno pode estar relacionado ao fato de a maioria dos entrevistados ser mulher, sendo este grupo já descrito pela literatura como consumidor de bebidas alcoólicas em menor proporção do que os homens. A experimentação do tabaco esteve presente em uma porcentagem considerável de estudantes, entretanto, poucos afirmaram consumo atual. Quanto aos transtornos mentais comuns, observou-se que um número expressivo apresentou respostas positivas para o grupo de sintomas "humor depressivo/ansioso". Os resultados obtidos permitiram ampliar os conhecimentos sobre a temática entre os universitários do curso de Enfermagem da Instituição estudada.

O rastreamento e monitoramento da ocorrência de uso de substâncias psicoativas lícitas e transtornos mentais comuns subsidiam informações importantes quanto à magnitude do fenômeno, no âmbito universitário. Sendo a universidade um ambiente privilegiado de discussão, onde todas as vertentes ideológicas possuem e devem ter voz, faz-se necessária a maior abordagem acadêmica, seja por meio da sua inserção de forma transversal na proposta curricular ou na realização de seminários com a participação de diversas áreas do conhecimento ou na realização de estudos sobre o uso problemático de álcool, consumo de tabaco e sofrimentos emocionais em estudantes universitários.

A comparação dos resultados possibilita o planejamento de programas de prevenção mais condizentes com as características e necessidade de cada comunidade, contribuindo para o desenvolvimento de estratégias preventivas e a busca por melhor qualidade de vida dos acadêmicos.

Como fator limitante deste e de outros estudos realizados com a população universitária, aponta-se o fato de abordar os participantes no ambiente escolar, uma vez que os estudantes podem se sentir constrangidos em descrever seu consumo de substâncias, mesmo sendo garantida tranquilidade para responder aos questionários e este ser informado sobre o sigilo das informações coletadas.

\section{Agradecimentos}

À Secretaria Nacional de Políticas sobre Drogas do Ministério da Justiça (SENADMJ) e à Escola de Enfermagem de Ribeirão da Universidade de São Paulo, Centro Colaborador da Organização Mundial da Saúde para o Desenvolvimento da Enfermagem, pela oportunidade de realizar este estudo e especializar-me em Álcool e outras Drogas Psicoativas.

\section{References}

1. Costa EFO, Santana YS, Santos ATRA, Martins LAN, Melo EV, Andrade TM. Sintomas depressivos entre internos de medicina em uma 
universidade pública brasileira. Rev Assoc Med Bras. 2012;58(1):53-9.

2. Furegato ARF, Santos JLF, Silva EC. Depressão entre estudantes de dois cursos de enfermagem: autoavaliação da saúde e fatores associados. Rev Bras Enferm. 2010 jul-ago;63(4):509-16.

3. Corradi-Webster CM, Esper LH, Pillon SC. A enfermagem e a prevenção do uso indevido de drogas entre adolescentes. Acta Paul Enferm. 2009;22(3):331-4.

4. Yosetake LL. Co-ocorrência de uso problemático de álcool, e transtorno mental comum em estudantes de graduação da área da saúde. [Dissertação de Mestrado]. Ribeirão Preto: Faculdade de Ciências Farmacêuticas de Ribeirão Preto da Universidade de São Paulo; 2007. $100 \mathrm{p}$.

5. Presidência da República (BR). Secretaria Nacional de Políticas sobre Drogas. I Levantamento nacional sobre o uso de álcool e outras drogas entre universitários das 27 capitais brasileiras. Brasília: Senad; 2010.

6. Franco GP, Barros ALPL, Nogueira-Martins LA. Qualidade de vida e sintomas depressivos em residentes de enfermagem. Rev. Latino-Am. Enfermagem. 2005;13(2):139-44.

7. Lima MCP, Domingues MS, Cerqueira ATAR. Prevalência e fatores de risco para transtornos mentais comuns entre estudantes de medicina. Rev Saúde Pública. 2006;40(6):1035-41.

8. Lucas ACS, Parente RCP, Picanço NS, Conceição DA, Costa KRC, Magalhães IRS, et al. Uso de psicotrópicos entre universitários da área da saúde da Universidade Federal do Amazonas, Brasil. Cad Saúde Pública. 2006;22(3):663-71.

9. Paduani GF, Barbosa GA, Morais JCR, Pereira JCP, Almeida MF, Prado MM, et al. Consumo de álcool e fumo entre os estudantes da Faculdade de Medicina da Universidade Federal de Uberlândia. Rev Bras Educ Médica. 2008;32(1):66-75.

10. Bourguignon LN, Silva, BP, Coelho MP, Siqueira MM. O uso do tabaco entre os estudantes de enfermagem do Centro Universitário do Espírito Santo (CEUNES). Rev Bras Pesqui Saúde 2011;13(4):35-40.

11. Matsumoto KS, Craveiro MF, Rocha PR, Lopes GT. O uso do tabaco entre os universitários de enfermagem da Universidade do estado do Rio de Janeiro (UERJ). SMAD, Rev. Eletrônica Saúde Mental Álcool Drog. (Ed. port.). [Internet]. 2005 [acesso 20 ago 2009]; 1(2). Disponível em: <http://www2.eerp.usp.br/ resmad/artigos/2005v1n2a05.pdf>

12. Portugal FB, Souza RS, Buaiz V, Siqueira MM. Uso de drogas por estudantes de Farmácia da Universidade Federal do Espírito Santo. J Bras Psiquiatr. 2008;57(2):127-32.

13. Teixeira RF, Souza RS, Buaiz V, Siqueira MM. Uso de substâncias psicoativas entre estudantes de Odontologia da Universidade Federal do Espírito Santo. Ciênc Saúde Coletiva. 2010;15(3):655-62.

14. Moretti-Pires RO; Corradi-Webster, CM. Adaptação e validação do Alcohol Use Disorder Identification Test (AUDIT) para população ribeirinha do interior da Amazônia, Brasil. Cad Saúde Pública. 2011;27:497-509.

15. Iacoponi E, Mari JJ. Reability and factor structure of the Portuguese version of Self Reporting Questionnaire. Int J Soc Psychiatry. 1988;3:213-22.

16. Ministério da Saúde (BR). Conselho Nacional de Saúde. Resolução No 466 de 12 de dezembro de 2012. Aprova as Diretrizes e normas regulamentadoras de pesquisas envolvendo seres humanos. Diário Oficial da União, Brasília, DF, 13 jun. 2013. Seção I, p. 59-62.

17. Silva BP. O Uso do Tabaco entre os Universitários de Enfermagem das Faculdades Unificadas Doctum, Campus Guarapari, Espírito Santo. [Trabalho de Conclusão de Curso]. Guarapari: Faculdades Unificadas Doctum; 2009.

18. Pillon SC, Santos SC, Gonçalves AMS, Araújo KM, Funai A. Fatores de risco, níveis de espiritualidade e uso de álcool em estudantes de dois cursos de enfermagem. SMAD, Rev. Eletrônica Saúde Mental Álcool Drog. (Ed. port.) [Internet]. 2010 [acesso 5 ago 2011]; 6, n.espec:493-513. Disponível em: <http://www2. eerp.usp.br/resmad/artigos/SMADv6nspeca08. pdf $>$.

19. Santos ML, Marcelino PW, Berardinelli LMM. Universidade, Jovens e Consumo de álcool: um estudo exploratório. Online Braz J Nurs [Internet]. 2012 abril; [acesso 14 maio 2012]; 11(1): Disponível em: http://www.objnursing. uff.br/index.php/nursing/article/view/3576.

Recebido: 10.12.2013 Aceito: 22.04.2014 